\title{
Sustained Release of Phosphates From Hydrogel Nanoparticles Suppresses Bacterial Collagenase and Biofilm Formation in vitro
}

\author{
Dylan Nichols ${ }^{1}$, Marja B. Pimentel ${ }^{1}$, Fernando T. P. Borges ${ }^{2}$, Sanjiv K. Hyoju ${ }^{3}$, \\ Fouad Teymour ${ }^{2}$, Seok Hoon Hong ${ }^{2}$, Olga Y. Zaborina ${ }^{3}$, John C. Alverdy ${ }^{3}$ and \\ Georgia Papavasiliou ${ }^{1 *}$
}

\begin{abstract}
${ }^{1}$ Department of Biomedical Engineering, Illinois Institute of Technology, Chicago, IL, United States, ${ }^{2}$ Department of Chemical and Biological Engineering, Illinois Institute of Technology, Chicago, IL, United States, ${ }^{3}$ Department of Surgery, University of Chicago, Chicago, IL, United States
\end{abstract}

\section{OPEN ACCESS}

Edited by:

Qingxin Mu,

University of Washington,

United States

Reviewed by:

Jianbo Jia,

Guangzhou University, China

Kusum Harjai,

Panjab University, India

*Correspondence: Georgia Papavasiliou

papavasiliou@iit.edu

Specialty section: This article was submitted to

Nanobiotechnology,

a section of the journal Frontiers in Bioengineering and

Biotechnology

Received: 23 February 2019 Accepted: 10 June 2019

Published: 26 June 2019

Citation:

Nichols D, Pimentel MB, Borges FTP

Hyoju SK, Teymour F, Hong SH,

Zaborina OY, Alverdy JC and

Papavasiliou G (2019) Sustained

Release of Phosphates From Hydrogel Nanoparticles Suppresses Bacterial Collagenase and Biofilm Formation

in vitro.

Front. Bioeng. Biotechnol. 7:153.

doi: 10.3389/fbioe.2019.00153
Intestinal disease or surgical intervention results in local changes in tissue and host-derived factors triggering bacterial virulence. A key phenotype involved in impaired tissue healing is increased bacterial collagenase expression which degrades intestinal collagen. Antibiotic administration is ineffective in addressing this issue as it inadvertently eliminates normal flora while allowing pathogenic bacteria to "bloom" and acquire antibiotic resistance. Compounds that could attenuate collagenase production while allowing commensal bacteria to proliferate normally would offer major advantages without the risk of the emergence of resistance. We have previously shown that intestinal phosphate depletion in the surgically stressed host is a major cue that triggers $P$. aeruginosa virulence which is suppressed under phosphate abundant conditions. Recent findings indicate that orally administered polyphosphate, hexametaphosphate, (PPi) suppresses collagenase, and biofilm production of $P$. aeruginosa and $S$. marcescens in animal models of intestinal injury but does not attenuate E. faecalis induced collagenolytic activity (Hyoju et al., 2017). Systemic administration of phosphates, however, is susceptible to rapid clearance. Given the diversity of collagenase producing bacteria and the variation of phosphate metabolism among microbial species, a combination therapy involving different phosphate compounds may be required to attenuate pathogenic phenotypes. To address these barriers, we present a drug delivery approach for sustained release of phosphates from poly(ethylene) glycol (PEG) hydrogel nanoparticles. The efficacy of monophosphate (Pi)- and PPi-loaded NPs (NP-Pi and NP-PPi, respectively) and a combination treatment (NP-Pi + NP-PPi) in mitigating collagenase and biofilm production of gram-positive and gram-negative pathogens expressing high collagenolytic activity was investigated. NP-PPi was found to significantly decrease collagenase and biofilm production of $S$. marcescens and $P$. aeruginosa. Treatment with either NP-Pi or NP-Pi + NP-PPi resulted in more prominent decreases in E. faecalis collagenase compared to NP-PPi alone. The combination treatment was also found to significantly reduce $P$. aeruginosa collagenase production. Finally, significant attenuation in biofilm dispersal was observed with NP-PPi or NP-Pi + NP-PPi treatment across all test pathogens. These findings 
suggest that sustained release of different forms of phosphate confers protection against gram-positive and gram-negative pathogens, thereby providing a promising treatment to attenuate expression of tissue-disruptive bacterial phenotypes without eradicating protective flora over the course of intestinal healing.

Keywords: polyphosphate, nanoparticles, hydrogel, microbes, collagenase, intestinal epithelium, biofilm, monophosphate

\section{INTRODUCTION}

The intestinal tract routinely undergoes a variety of injuries due to disease or direct surgical manipulation. The process by which successful repair and return of function occurs following these insults is highly dependent on the composition and function of the intestinal microbiota. Consequently, wound healing, and repair of the intestinal tract is a complex process due to the presence of intestinal microbiota, which can either enhance or severely impair the healing process. For example, severe persistent inflammation in diseases such as ulcerative colitis and Crohn's disease (i.e., inflammatory bowel disease) are recognized to be due to loss of the normal microbiome and its replacement by pathogens. In addition, iatrogenic injury, such as that which occurs when gastroenterologists remove intestinal tumors via endoscopy or major surgical resection, may also disrupt the normal microbiome leaving a large wound to heal in the presence of highly pathogenic bacteria. These curative surgical interventions are often complicated by excessive scar formation, stricture, stenosis, or grossly inadequate healing. In extreme cases, this can lead to perforation of the intestine, resulting in peritonitis and sepsis.

Previous studies indicate that certain bacteria (i.e., E. faecalis, S. marcescens, $P$. aeruginosa) are capable of secreting elevated levels of collagenase, resulting in collagen degradation (Zaborin et al., 2009, 2012; Shogan et al., 2015). The presence of this phenotype in intestinal tissue leads to excessive scar formation and healing impairment. In our previous work we identified that E. faecalis, a normal inhabitant of the intestinal microbiota, may be provoked to express enhanced collagenase in the gut during surgical injury, leading to major post-operative complications including anastomotic leak (Shogan et al., 2015). Using a clinically relevant mouse model of anastomotic leak, we demonstrated that exposure to pre-operative radiation followed by distal colon resection and intestinal inoculation with $P$. aeruginosa, a pathogen commonly found in the radiated intestine, results in significant incidence of leak as compared to radiated tissues alone (Valuckaite et al., 2009). P. aeruginosa strains retrieved from leaking anastomotic tissues were also shown to shift to a destructive phenotype (increased levels of pyocyanin and collagenase) (Olivas et al., 2012). Additional problematic pathogens such as $S$. marcescens also produce collagenases to disrupt intestinal healing (Olivas et al., 2012; Hyoju et al., 2017).

A common approach to address these issues is administration of antibiotics which indiscriminately disrupts normal intestinal microflora which have been shown to be beneficial to healing (Carlet, 2012). Clinical studies indicate that patients remain colonized by strains of $E$. faecalis and $P$. aeruginosa for as long as 1 week after surgery even after receiving oral and intravenous antibiotic administration (Ohigashi et al., 2013). This may be due, in part, to their multi-drug resistant nature as the promiscuous use of antibiotics remains a problem (Buffie and Pamer, 2013). Thus, there is an urgent need for development of non-antibiotic compounds that do not disrupt the normal intestinal microbial community structure. Normal flora protect against overgrowth and invasion of pathogens by a process of competitive exclusion and by suppressing key pathogenic traits among bacteria. One such approach is to create a nutrient rich microenvironment at the site of injury to suppress virulence expression.

Previously, we have demonstrated that phosphate-based therapy is a promising therapeutic approach for suppression of virulent expressing phenotypes since inorganic phosphate abundance is a key factor involved in attenuation of quorum sensing and global virulence (Zaborin et al., 2009). Furthermore, we have demonstrated that depletion of extracellular phosphate occurs in the intestinal tract following surgical injury triggering bacterial virulence (Zaborin et al., 2009, 2012). As a result of extracellular phosphate depletion, pathogens scavenge phosphate from host tissues, leading to disruption of intestinal mucus. Our in vivo and in vitro findings indicate that replenishment of monophosphate $(\mathrm{Pi})$ in the intestinal epithelium via oral administration prevents virulence expression while maintaining bacterial survival (Zaborin et al., 2009). Polyphosphate, a polymer of phosphate residues linked by high-energy phosphoanhydride bonds as in ATP, also plays a key mechanistic role in survival and virulence across a broad range of bacteria (Rao et al., 2009). Our recent findings indicate free PPi decreases in vitro collagenase and biofilm production of gram-negative $P$. aeruginosa and $S$. marcescens and that oral administration of PPi prevents anastomotic leak in mice subjected to intestinal injury and inoculation with these pathogens (Hyoju et al., 2017). Conversely, PPi treatment did not demonstrate significant collagenase attenuation of gram-positive E. faecalis or prevent E. faecalis-induced anastomotic leak (Hyoju et al., 2017). Given the diversity of bacteria that produce collagenase and the variation in phosphate metabolism among microbial species, a combination therapy involving different phosphate compounds may be required to attenuate the expression of pathogenic phenotypes. In addition, oral administration of inorganic phosphate is prone to loss of bioavailability in the colon, a common site of surgery to remove cancerous tumors. Inorganic phosphate is rapidly absorbed in the small intestine, and as a result, high concentrations of orally administered phosphates are required to increase phosphate concentration which may impair kidney function. 
To address these barriers, we present a drug delivery approach that provides sustained release of phosphates from hydrogel nanoparticles to attenuate collagenase and biofilm production across gram-negative and gram-positive pathogens. Successful in vitro demonstration of this approach holds promise for in vivo replenishment of phosphate levels in intestinal tissue. In previous work we developed a novel process to produce crosslinked hydrogel nanoparticles of poly(ethylene) glycol (NP) for loading of hydrophilic therapeutics, including phosphates, enabling their sustained release (Vadlamudi et al., 2019). We have shown that this process results in the production of phosphate as well as polyphosphate loaded NPs (NP-Pi and $\mathrm{NP}-\mathrm{PPi}$, respectively) and in sustained release of $\mathrm{Pi}$ and $\mathrm{PPi}$ (Yin et al., 2017). Importantly, our published findings indicate that NP-PPi attenuate $P$. aeruginosa pyoverdine production in a dose-dependent manner and significantly decrease pyocyanin production while maintaining bacterial survival (Yin et al., 2017). The goal of this study is to determine the effectiveness of NP$\mathrm{PPi}$ and a combination treatment of NP-Pi and NP-PPi (NP$\mathrm{Pi}+\mathrm{NP}-\mathrm{PPi}$ ) for suppression of collagenase and biofilm across gram-positive and gram-negative pathogens. In this work we evaluate the minimal inhibitory concentration of the phosphateloaded nanoparticle formulations and screen their efficacy in suppressing in vitro collagenase and biofilm production for three key pathogens (P. aeruginosa, S. marcescens, and E. faecalis) identified to express high collagenolytic activity and to induce anastomotic leak in both rodents and humans.

\section{MATERIALS AND METHODS}

\section{Materials}

Poly(ethylene glycol) diacrylate (PEGDA, MW = $575 \mathrm{Da}$ ), $\mathrm{N}$-vinyl pyrrolidone (NVP), potassium phosphate monobasic (phosphate, Pi), sodium hexametaphosphate (polyphosphate, PPi), potassium persulfate (KPS), sorbitan monooleate (SPAN80), PEG (20) sorbitan monolaurate (TWEEN20), cyclohexane, and acetone were purchased from Sigma-Aldrich (St. Louis, MO, USA). Ultra-high purity (UHP) nitrogen was purchased from Airgas (Radnor, PA, USA).

Monomethyl ether hydroquinone (MEHQ) inhibitor (400$600 \mathrm{ppm}$ ) was removed from the PEGDA monomer by passing the solution through a prepacked column (Sigma Aldrich). Sodium hydroxide inhibitor was removed from the NVP monomer by passing the solution through a $0.2 \mu \mathrm{m}$ syringe filter (Millipore, Burlington, MA, USA). All other materials were used as received.

\section{Nanoparticle Synthesis, Purification, Phosphate, and Polyphosphate Loading}

Hydrogel nanoparticles were created using an inverse phase miniemulsion polymerization process we have formerly developed for encapsulation of monophosphate (i.e., potassium monobasic phosphate) but with slight modifications as described herein (Yin et al., 2017; Vadlamudi et al., 2019). The nanoparticle synthesis process requires the formation of a lipophobe, typically an inorganic salt (i.e., phosphate), for the prevention of Ostwald ripening (Vadlamudi et al., 2019).
To achieve sufficient loading of polyphosphate (PPi) within the hydrogel nanoparticles, potassium monobasic phosphate (monophosphate) loaded nanoparticles were first created using inverse phase miniemulsion polymerization, purified of excess reactants, rinsed to remove monophosphate, and post-loaded with PPi (as described below). This enabled loading of a desired $\mathrm{PPi}$ concentration post nanoparticle synthesis. Monophosphate (Pi)-loaded nanoparticles (NP-Pi) were also synthesized as previously described (Yin et al., 2017; Vadlamudi et al., 2019). The polymerization reaction was carried out in an inverse miniemulsion created through emulsification of an aqueous phase precursor into an organic phase (Vadlamudi et al., 2019). The aqueous phase consisted of a precursor solution containing a PEG diacrylate (PEGDA) crosslinker, N-vinyl pyrrolidone (NVP), sorbitan monolaurate (TWEEN 20) as the water-soluble surfactant, potassium persulfate (KPS) as the thermal initiator and potassium monobasic phosphate (monophosphate) as the osmotic pressure agent in deionized (DI) water. The total concentration of cross-linkable double bonds in the precursor was fixed at $1.36 \mathrm{M}$ and the initiator concentration was set as 1.5 $\%$ molar ratio of initiator to reactive double bonds. The organic phase contained cyclohexane and sorbitan monooleate (SPAN 80 ) as the organic soluble co-surfactant.

The inverse miniemulsion was generated using a two-step homogenization process. The organic phase was chilled in an ice bath and then homogenized using a rotor-stator homogenizer (10 mm saw-tooth generator, IPRO 250, Pro-Scientific) at 20,000 $\mathrm{rpm}$. The aqueous phase was added drop-wise to the organic phase and homogenization continued for $15 \mathrm{~min}$. The resulting emulsion was exposed to high energy sonication using an ultrasonic horn (SONICS Vibracell VCX 750 Watts) at $90 \%$ amplitude for five pulses of $1 \mathrm{~min}$ each. The stable inverse miniemulsion was transferred to a sealed glass batch reactor, kept under a nitrogen blanket and continuously sparged with pure nitrogen for $1 \mathrm{~h}$. When sparging was stopped the reactor was partially immersed in an oil bath set to achieve a reaction temperature of $56 \pm 0.5^{\circ} \mathrm{C}$. The reaction proceeded at this temperature for $4.5 \mathrm{~h}$ after which the resulting stable nanoparticle suspension was removed from the reactor and allowed to cool in a glass jar at room temperature.

PEG hydrogel nanoparticles were precipitated from the colloidal suspension by the addition of $70-80 \mathrm{~mL}$ of acetone. Agglomerated particles were allowed to settle overnight at $4{ }^{\circ} \mathrm{C}$ after which two layers were formed, a white bottom layer and yellowish supernatant. The supernatant was removed by decantation followed by addition of $120 \mathrm{~mL}$ of fresh acetone and re-suspension by sonication at $90 \%$ amplitude. The suspension was centrifuged at $3,220 \mathrm{~g}$ at $4^{\circ} \mathrm{C}$ for $90 \mathrm{~min}$ followed by removal of the supernatant and replacement with fresh acetone. This sonication and centrifugation cycle was completed twice more to ensure removal of residual cyclohexane and surfactant. Rinsed nanoparticle pellets were dried under vacuum for $48 \mathrm{~h}$. Dried nanoparticle agglomerates were transferred to a mortar and crushed with a pestle into a fine powder, yielding monophosphate loaded nanoparticles (NP-Pi).

To remove encapsulated monophosphate, nanoparticles were rinsed with DI water, sonicated, swollen in DI water for $2 \mathrm{~h}$, 
followed by centrifugation at $3,220 \mathrm{~g}$ and $4^{\circ} \mathrm{C}$ for $30 \mathrm{~min}$ and removal of supernatant. This process was repeated eight times to ensure complete removal of the monophosphate from the nanoparticles (confirmed by measuring the amount of phosphate release using the molybdenum blue method as described below). Fully rinsed nanoparticles devoid of phosphate (blank nanoparticles) were used as controls in all experiments.

Post-loading of heat treated PPi $\left(56^{\circ} \mathrm{C}\right.$ for $\left.4.5 \mathrm{~h}\right)$ (Yin et al., 2017) into blank NPs was achieved by suspending 1.5 grams of blank NPs in $40 \mathrm{~mL}$ of a $150 \mathrm{mg} / \mathrm{mL}$ solution of sodium hexametaphosphate $(\mathrm{PPi})$. The solution was sonicated at $15 \%$ amplitude for $5 \mathrm{~s}$ and stored at $37^{\circ} \mathrm{C}$ for $72 \mathrm{~h}$. The nanoparticles were then centrifuged at $3,220 \mathrm{~g}$ and $4^{\circ} \mathrm{C}$ for $30 \mathrm{~min}$ and the supernatant was removed. To remove excess $\mathrm{PPi}$ from the particle surface, nanoparticles were rinsed with $5 \mathrm{~mL}$ DI water, centrifuged, and the supernatant removed. Polyphosphate loaded nanoparticles (NP-PPi) were frozen at $-80^{\circ} \mathrm{C}$, lyophilized, crushed with a pestle into a fine powder, and stored dry at room temperature until use.

\section{Nanoparticle Characterization}

Three types of nanoparticles were used in this study: sodium monophosphate loaded NPs (NP-Pi), blank NPs (formed by rinsing NP-Pi to remove $\mathrm{Pi}$ ), and sodium hexametaphosphateloaded nanoparticles (NP-PPi), formed from blank NPs subsequently post-loaded with PPi. All nanoparticles were synthesized as three separate batches and particle size distribution, diameter, $\zeta$-potential, and swelling ratio were obtained as an average from all batches. Particle size and $\zeta$-potential measurements were performed using a $0.1 \mathrm{mg} / \mathrm{mL}$ nanoparticle concentration. Particle size distribution was quantified using a nanosizer (NanoSight LM10, Malvern, UK) equipped with nanoparticle tracking analysis software (NanoTracking v 3.0). Zeta ( $\zeta$-) potential was determined using dynamic light scattering (DLS) (Zetasizer Nano ZS, Malvern, UK) with measurements made in a $10 \mathrm{mM} \mathrm{NaCl}$ solution. The mass swelling ratio of hydrogel nanoparticles was determined by gravimetric analysis and calculated from the ratio of the swollen (in PBS) to dry nanoparticle weights. All values were obtained as averages of at least three independent measurements. SEM imaging was used to visualize the shape and formation of blank (NP) and PPi-loaded nanoparticles (NP-PPi) in the dehydrated (dried) state. Briefly, nanoparticles were dissolved in $99 \%$ ethyl alcohol to a concentration of $1 \mathrm{mg} / \mathrm{mL}$. The solution was vortexed and sonicated for mixing. A metal support stub was covered with round carbon tape adhesives onto which the solution was pipetted. Stubs containing NP or NP-PPi were left under the hood until all the ethanol evaporated. Stubs were then imaged using an JEOL JSM 6701-F Field Emission Scanning Electron Microscope and images of the nanoparticles acquired.

\section{Quantification of Polyphosphate Release Kinetics From NP-PPi}

Polyphosphate release from nanoparticles was measured via a molybdenum blue absorbance assay at $340 \mathrm{~nm}$ (Phosphorus Liqui-UV No. 0830, StanBio Laboratory, USA). A standard curve was created for PPi (heat treated) in tryptone yeast (TY) media using the molybdenum blue assay kit. Nanoparticles were incubated in TY media $(18 \mathrm{mg} / \mathrm{mL})$ at $37^{\circ} \mathrm{C}$ over $72 \mathrm{~h}$. PPi released from nanoparticles was measured under near perfect sink conditions at several pre-determined times. Specifically, nanoparticles were centrifuged at $6,000 \mathrm{~g}$ for $3 \mathrm{~min}$, followed by removal of the supernatant $(1 \mathrm{~mL})$ and replacement with fresh TY media. Release kinetics of Pi from NP-Pi have been reported in our prior studies (Yin et al., 2017).

\section{Collagenolytic Bacterial Strains}

The commercially available constitutive bioluminescent $P$. aeruginosa PAO1 derivative XEN41 strain with the luxCDABE cassette incorporated into chromosome (Calpier Life Sciences, Inc), was used in these studies. This strain was previously confirmed in mouse model experiments (Fink et al., 2011) to display a highly collagenolytic P2 phenotype (Luong et al., 2014). S. marcescens ICU2-4 strain was isolated from an ICU patient at the University of Chicago (Zaborin et al., 2014) and was characterized as collagenolytic (Hyoju et al., 2017). A bioluminescent strain ICU2-4lum for S. marcescens was created by transformation with the pAKlux2 plasmid containing the luxCDABE luciferase cassette (Addgene, Ref \#14080) (Karsi and Lawrence, 2007). The antibiotic resistant strain E. faecalis E61 was isolated from rat anastomotic tissue and was characterized as collagenolytic (Shakhsheer et al., 2016). All bacterial strains were stored in $10 \%$ glycerol stock at $-80^{\circ} \mathrm{C}$. Cells from frozen stocks were plated onto tryptone yeast (TY) plates and grown overnight at $37^{\circ} \mathrm{C}$. An inoculation loop of plated bacteria was transferred to culture tubes containing $2 \mathrm{~mL}$ of TY media that was subsequently cultured overnight at $37^{\circ} \mathrm{C}$. In all experiments, bacterial strains were utilized from these liquid cultures and diluted 1:100 in fresh TY media.

\section{Minimal Inhibitory Concentration of NP-Pi, NP-PPi, and Mixtures of NP-Pi + NP-PPi}

The minimal inhibitory concentration (MIC) of nanoparticle formulations [concentration range between 1 and $10 \%$ (w/volume)] was determined for the three pathogens. In the case of gram-negative pathogens, an MIC screening was conducted for NP-PPi and a mixture of NP-Pi + NP-PPi since our prior findings indicated that monophosphate (Pi) treatment (free $\mathrm{Pi}$ or NP-Pi) does not attenuate collagenase production for these pathogens (data not shown). The total concentration of nanoparticles used was kept constant among all treatments. In the case of E. faecalis, an additional treatment group, NP-Pi, was also explored as monophosphate was previously shown to attenuate its collagenolytic activity. Six different NP treatment concentrations were tested $[1,2,4,6,8$, and $10 \%(\mathrm{w} / \mathrm{v})]$ for each pathogen in addition to the no treatment control. To determine the MIC values of each formulation for each pathogen, overnight cultures were diluted 1:100 in TY medium, inoculated with the specified treatment concentration and incubated at $37^{\circ} \mathrm{C}$. CFUs were then quantified for each group and the MIC value was determined based on the lowest nanoparticle concentration at which statistical inhibition in growth was noted. 


\section{Evaluation of Bacterial Collagenolytic Activity}

Bacterial collagenolytic activity was evaluated in the absence and presence of monophosphate and polyphosphate treatment. Treatment groups included free PPi (heat-treated), blank nanoparticles (NP), NP-Pi, NP-PPi, and mixtures of NP-Pi and NP-PPi (NP-Pi + NP-PPi). Collagenase levels were quantified using a collagenase assay kit $\left(\mathrm{DQ}^{\mathrm{TM}}\right.$ gelatin from pig skin, fluorescein conjugate, Invitrogen, Eugene, OR). Bacterial strains were grown overnight in liquid TY media and then diluted 100 -fold. A volume of $190 \mu \mathrm{l}$ of diluted bacteria in liquid TY, with or without PPi treatment, was added to $10 \mu \mathrm{l}$ of collagen substrate $(1 \mathrm{mg} / \mathrm{mL}$; DQ gelatin from pig skin, fluorescein conjugate). The negative control consisted of 190 $\mu \mathrm{l}$ TY media with $10 \mu \mathrm{l}$ of collagen substrate. The result of the reaction was measured at the appropriate time points with fluorescence excitation and emission at 485 and $528 \mathrm{~nm}$, respectively, using a microplate reader (FL x800, Bio-Tek Instruments Inc). Values obtained for negative controls were subtracted from experimental treatment groups to account for background fluorescence. All experiments were carried out in triplicate. At each time point, the luminescence of each sample was measured and normalized to the number of bacteria in each sample.

\section{Bacterial Adherence/Biofilm Assay}

Overnight culture of bacterial strains was diluted 1:100 in 500 $\mu l$ TY media. The OD of 1:100 dilutions of overnight cultures was in the range of $0.02-0.05(\mathrm{OD}=600 \mathrm{~nm})$ Depending on the group (with or without nanoparticle treatment) nanoparticles were added to the bacterial solution right before being aliquoted into 96-well polystyrene plate. Plates containing $200 \mu$ l per well of this solution were incubated at $37^{\circ} \mathrm{C}$ for $24 \mathrm{~h}$ and further accessed for biofilm formation via crystal violet staining (Shakhsheer et al., 2016). Briefly, after incubation the medium was removed from the plates and used for quantifying CFUs for all bacterial strains. Plates were gently washed three times with DI water and allowed to dry. Two hundred $\mu \mathrm{l}$ of $0.1 \%$ crystal violet was added to each well at room temperature. After $20 \mathrm{~min}$ the stain was removed, and the plates gently washed three times with DI water. After drying, $200 \mu$ l of ethanol (95\%) was added to solubilize the crystal violet stained in each well and the absorbance was then measured at $590 \mathrm{~nm}$ and normalized to growth.

\section{Quantification of Bacterial Growth}

Optical density measurements are commonly used to normalize collagenase and adherence values to the number of bacteria in each sample; however, the presence of nanoparticles in culture interferes with the accuracy of optical density measurements for quantifying bacterial growth. To address this issue, we used bioluminescent bacterial strains for $P$. aeruginosa and $S$. marcescens as described above to quantify growth via luminescence. Comparisons of luminescence to CFUs were made to ensure referenceable values for bacterial growth. This was achieved by evaluating luminescence and plating for CFUs at different time points. A standard curve of luminescence and CFUs was then created allowing for quantification of an "effective" CFU value for each observed luminescent value. This technique was used for bioluminescent gram-negative bacterial strains. Standard curves for luminescence and CFUs are provided as supplementary data (Figures S1, S2). In the case of E. faecalis, bacterial growth was measured by counting colony forming units (CFUs).

\section{Statistical Analysis}

Each experiment was performed in triplicate unless otherwise noted. Quantitative results are presented as the mean, plus, and minus the standard deviation. Statistical comparisons were performed using analysis of variance (ANOVA) followed by a Holm-Sidak test for comparisons of data pairs. Statistical significance was considered for $p<0.05$.

\section{RESULTS AND DISCUSSION}

\section{Nanoparticle Characterization}

The in vitro effectiveness of phosphate-loaded NPs in attenuating collagenase and biofilm formation was evaluated against a control of blank nanoparticles (NPs) devoid of Pi or PPi. Since variations in particle physical properties have been shown to influence cell-nanoparticle interactions, and to discern whether the observed in vitro findings are mainly attributable to Pi or PPi treatment, we first characterized the physicochemical properties of blank NPs, NP-Pi, and NP post-loaded with PPi (NP-PPi). All nanoparticles were characterized in terms of hydrated particle size distribution, gravimetric swelling ratio (inversely related to the crosslink density of the nanoparticle network), and $\zeta$ potential (an indicator of particle surface charge characteristics). The values of each of these properties are reported in Table 1 and represent the average of three separate nanoparticle synthesis batches for each particle type. As expected, the mean diameter was found to be similar for NP, NP-PPi and NP-Pi ( $181 \pm 57$, $197 \pm 63 \mathrm{~nm}, 181 \pm 57$, respectively) with uniform particle size distribution as quantified by nanoparticle tracking analysis (Figures 1a,b). The swelling ratios of NP and NP-Pi $(4.26 \pm 0.26)$ and NP-PPi $(4.05 \pm 0.22)$ were similar as these particles were synthesized using identical polymerization conditions resulting in similar network structure (i.e., crosslink density) prior to postloading with PPi. In addition, blank NPs were synthesized by first forming NP-Pi followed by particle rinsing to remove Pi. The $\zeta$-potentials of NP-Pi and NP-PPi were found to be more negative $(-17.92 \pm 1.05 \mathrm{mV}$ and $-14.27 \pm 5.34 \mathrm{mV}$, respectively) as compared to $\mathrm{NP}(-11.80 \pm 5.27)$ which is expected due to the presence of phosphate ions in the former case. The $\zeta$ potential of NP-Pi was slightly more negative as compared to NP-PPi which may be attributed to the increased diffusivity and accumulation of monophosphate toward the nanoparticle surface as compared to PPi. Conductivity of all NP formulations was consistently between 1.0 and $1.1 \mathrm{mS} / \mathrm{cm}$. Collectively, the data suggest that the nanoparticles imbibe roughly four times their dry weight in aqueous solution and that they possess negative surface charge characteristics. Negative surface charge is desired for our intended application as it minimizes nanoparticle cellular uptake since the goal is to provide sustained release of phosphates extracellularly. Importantly, the data indicate no significant 
TABLE 1 | Particle diameter, swelling ratio, and $\zeta$-potential for blank and PPi-loaded hydrogel nanoparticles.

\begin{tabular}{lccc}
\hline & $\begin{array}{c}\text { Nanoparticle } \\
\text { diameter }(\mathbf{n m})\end{array}$ & $\begin{array}{c}\text { Mass swelling } \\
\text { ratio }\end{array}$ & $\begin{array}{c}\text { Zeta potential } \\
\mathbf{( m V})\end{array}$ \\
\hline NP (blank) & $181 \pm 57$ & $4.26 \pm 0.16$ & $-11.80 \pm 5.27$ \\
NP-PPi & $197 \pm 63$ & $4.05 \pm 0.22$ & $-14.27 \pm 5.34$ \\
NP-Pi & $181 \pm 57$ & $4.26 \pm 0.16$ & $-17.92 \pm 1.05$ \\
\hline
\end{tabular}

differences between NP, NP-Pi, and NP-PPi physicochemical properties, which allows us to attribute the observed in vitro responses to PPi release. SEM imaging was used to further verify the formation and visualize the shape of the resultant nanoparticles. Figures 1c,d provides representative SEM images of blank NPs (devoid of phosphate) and NP-PPi, respectively, in the dehydrated state. SEM imaging confirms similar nanoparticle morphology, shape and diameter in the presence or absence of $\mathrm{Pi}$ or PPi loading. A spherical particle morphology and diameter $<100 \mathrm{~nm}$ can be discerned from each of the acquired SEM images of nanoparticles in the dehydrated state. The nanoparticle diameters are expected as to be lower than those of the fully swollen state, the latter quantified using nanoparticle tracking analysis.

\section{Release Kinetics of Polyphosphate From NP-PPi}

Release of polyphosphate from NP-PPi was analyzed using a phosphorus absorbance assay kit (StanBio Labs, Boerne, Texas). A polyphosphate standard curve was generated for absorbance measurements with a PPi solution in tryptone yeast (TY) media, which was the media used during in vitro experiments with the different bacterial strains. The standard curve was linear in the range of $0.1-12.5 \mathrm{mg} / \mathrm{mL} \mathrm{PPi}$. Polyphosphate release from NP-PPi was measured in TY media at pre-determined times until PPi release could no longer be detected. Figure 2 displays the concentration of PPi released at specified times (refreshed release) as well as the cumulative release under near perfect sink conditions. The data shown in Figure 2 indicate an initial burst of PPi over the first hour with sustained release after that time until $72 \mathrm{~h}$. The cumulative release maximum was estimated to be $7.0 \mathrm{mg} / \mathrm{mL}$ PPi which represents the total amount of PPi released from the $18 \mathrm{mg} / \mathrm{mL}$ nanoparticle sample. To determine an "equivalent free PPi concentration" to use for in vitro experiments, release of PPi in TY media over the 72-h time period was measured for different NP-PPi concentrations. The measured absorbance was then converted to the ratio of PPi concentration over the initial concentration of NP-PPi. These concentrations allowed us to estimate an average equivalent polyphosphate ratio of $37 \pm 0.01 \% \mathrm{PPi}$. Figure 2 displays the PPi release for one representative batch of nanoparticles. However, two subsequent batches yielded similar release profiles and equivalent PPi concentrations. Thus, the equivalent free PPi concentration that was used in subsequent in vitro studies was estimated as $37 \%$ of the NP-PPi concentration. The theoretical maximum concentration of PPi within the nanoparticles was calculated as the equilibrium concentration after diffusion of the PPi post-loading solution into the nanoparticle load. This calculation yielded a maximum PPi concentration of $36 \%$ of the NP-PPi weight. Accounting for error, this would indicate a loading efficiency of nearly $100 \%$. The release data also suggest that the network swelling ratio and crosslink density is sufficient for post-loading within and subsequently releasing PPi from the hydrogel nanoparticles.

\section{Effect of Free PPi on Bacterial Collagenase Activity}

We have previously shown that oral administration of free PPi is effective at suppressing collagenase production and preventing anastomotic leak of gram-negative $S$. marcescens and $P$. aeruginosa (Hyoju et al., 2017). Free PPi treatment was not, however, found to be effective for E. faecalis (Hyoju et al., 2017). Since clinical studies have shown that patients remain colonized with pathogenic strains as long as 7 days post-surgery (Ohigashi et al., 2013), nanoparticles providing sustained release of PPi over this duration offer a promising drug delivery strategy for attenuation of collagenase activity. The ability of NP-PPi or NP-Pi + NP-PPi combinations to suppress collagenase and biofilm formation in vitro has not been previously explored and was evaluated for three different pathogens exhibiting high collagenolytic activity ( $P$. aeruginosa, $S$. marcescens, and E. faecalis).

To determine a concentration of NP-PPi to be used for in vitro studies, we first conducted dose-response studies of collagenase attenuation with free PPi for all three test pathogens. Concentrations of PPi tested ranged from 0.1 to $160 \mathrm{mM}$ (Figure 3). This range was chosen since microorganisms can synthesize polyphosphates at concentrations as high as $200 \mathrm{mM}$ (Harold, 1966) which is dramatically higher as compared to reported concentrations in animal tissue ( $\mu \mathrm{M}$ concentration) (Kumble and Kornberg, 1995; Lorenz et al., 1997). Collagenase activity and optical density were also measured after 8 hours of growth for gram-negative pathogens ( $P$. aeruginosa and $S$. marcescens) and after $16 \mathrm{~h}$ of growth for gram-positive $E$. faecalis. These two time points were chosen as we observed that $8 \mathrm{~h}$ of growth for both $P$. aeruginosa and S. marcescens and $16 \mathrm{~h}$ for E. faeclais were sufficient to "stress" the cultures under nutrient depletion. This induced "stress" can be noted by the high collagenase activity displayed at the 8 - and 16 -h time points. Figure 3 shows the effect of free PPi concentration on normalized collagenase levels for each pathogen. At PPi concentrations greater or equal to $2 \mathrm{mM}$, significant attenuation in $P$. aeruginosa collagenase activity is observed while PPi concentrations greater or equal to $0.1 \mathrm{mM}$ were found to be effective for suppression of $S$. marcescens collagenolytic activity. No discernable response to PPi treatment was observed for E. faecalis, although a reduction in collagenase was noted at a PPi concentration of $160 \mathrm{mM}$. This decrease, however, was not found to be statistically significant from the no treatment control. Based on these results, $6 \mathrm{mM}$ PPi was chosen as an effective dose for targeting both $P$. aeruginosa and $S$. marcescens collagenase attenuation. This target concentration was chosen because as it was greater than the minimum dose found to attenuate collagenase expression in the gram-negative test pathogens (2 $\mathrm{mM} \mathrm{PPi}$ ). In addition, $6 \mathrm{mM} \mathrm{PPi}$ corresponds 

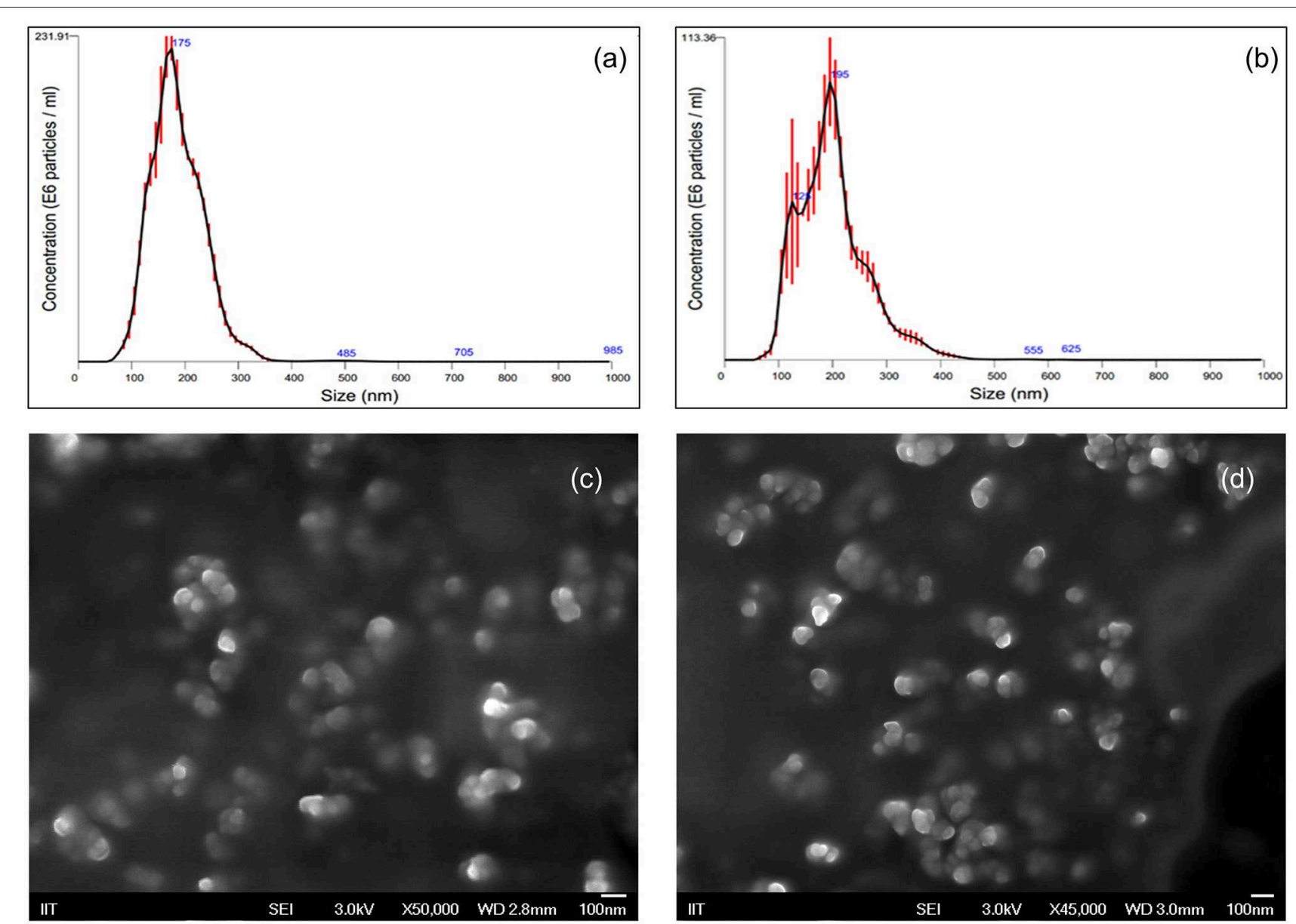

FIGURE 1 | Nanoparticle Characterization using Nanoparticle Tracking Analysis and Scanning Electron Microscopy. Particle size distributions for (a) blank NP (D10: $116.5 \mathrm{~nm}$, D50: $170.6 \mathrm{~nm}$, D90: $239.5 \mathrm{~nm}$ ) and (b) NP-PPi (D10: $113.4 \mathrm{~nm}$, D50: $184.2 \mathrm{~nm}$, D90: $276.1 \mathrm{~nm}$ ). Red error bars indicate \pm 1 standard error of the mean from three separate nanoparticle tracking measurements. SEM representative images of (c) blank NPs and (d) NP-PPi in the dehydrated state.

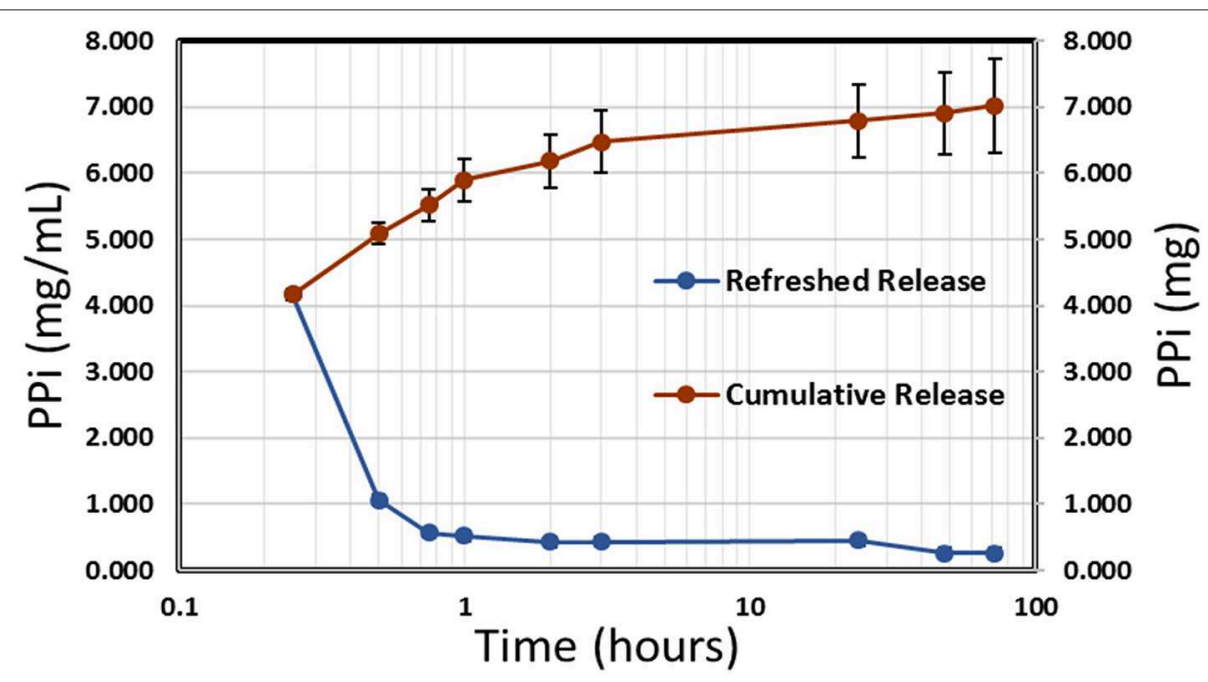

FIGURE 2 | Release kinetics of PPi from NP-PPi in TY media. Refreshed release indicates single time-point measurements (left vertical axis) and cumulative release indicates the sum of all previous single measurements (right vertical axis). 

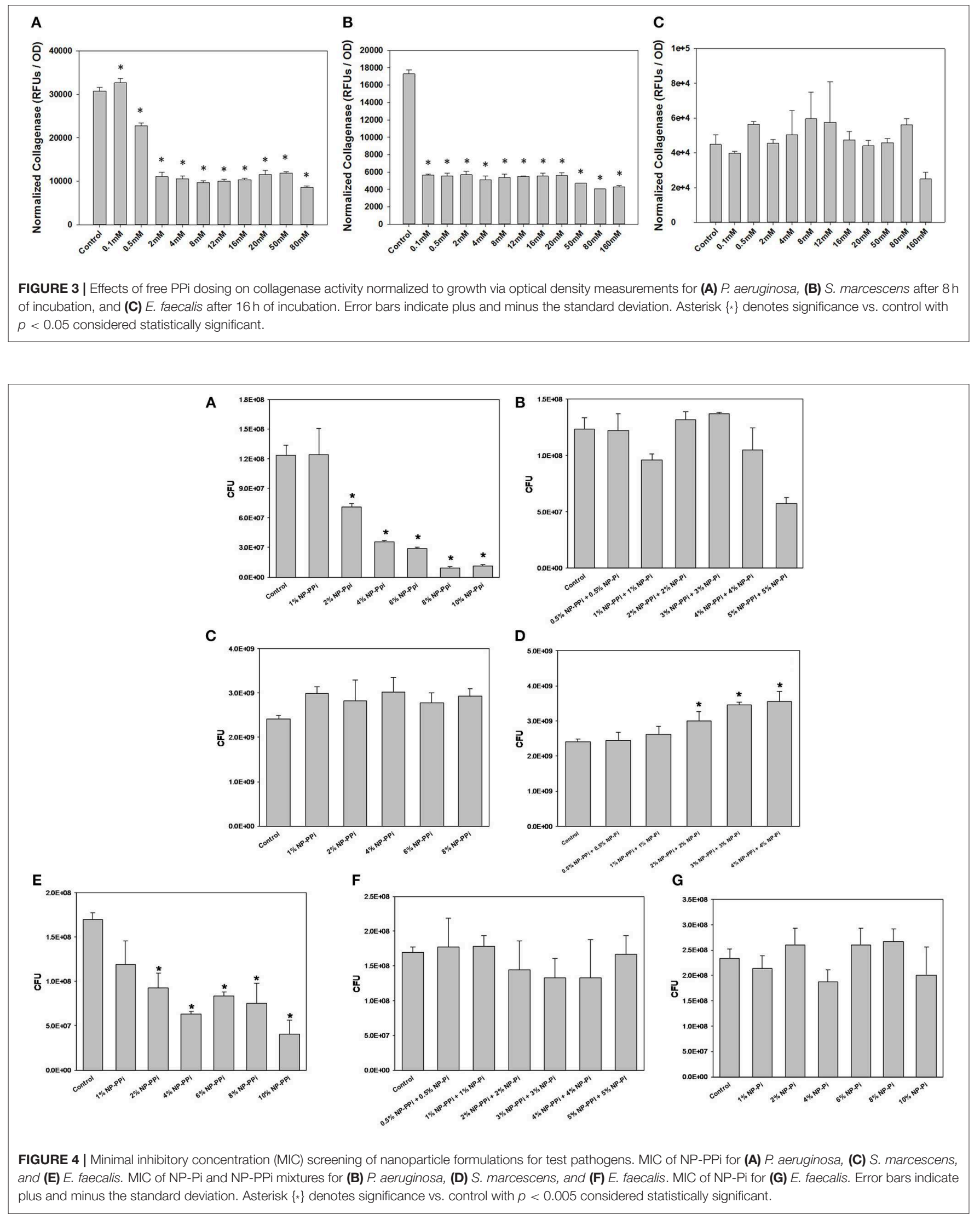
to a concentration of $1 \%$ (weight per volume) NP-PPi which was found to be below the MIC value of the gram-negative and gram- positive test pathogens (Figure 4). A concentration of $6 \mathrm{mM}$ free PPi corresponds to an equivalent concentration of $3.7 \mathrm{mg} / \mathrm{mL}(0.37 \% \mathrm{w} / \mathrm{vol})$ which was subsequently used in culture studies.

\section{Bactericidal Activity of Nanoparticles}

The minimum inhibitory concentrations (MIC) of phosphateloaded nanoparticle formulations were tested within the range of $1-10 \%$ nanoparticles (w/vol). This chosen range of nanoparticle concentration also corresponds to 6-60 mM free PPi which was found to be effective at attenuating collagenase levels of the gramnegative test pathogens. Since previous studies (Hyoju et al., 2017) and the results from Figure 3C indicated that free PPi was not effective at attenuating E. faecalis collagenase production, we hypothesized that NP-Pi or a combination treatment of NP-Pi + NP-PPi would be more effective, the latter beneficial for attenuating collagenase across multiple pathogens. An MIC screening for NP-PPi and NP-Pi + NP-PPi was conducted in the case of $P$. aeruginosa and $S$. marcescens. The MIC value was taken as the minimum concentration that resulted in statistical significance in reduction in CFU as compared to the no treatment control. As shown in Figure 4A, the MIC for NP-PPi was $2 \%(\mathrm{w} / \mathrm{vol})$ in the case of $P$. aeruginosa between the tested range of $1-10 \%(\mathrm{w} / \mathrm{vol})$ NP-PPi. No bactericidal effects were observed with NP-Pi + NP-PPi treatment for $P$. aeruginosa (Figure 4B). In addition, no bactericidal effects were for NP-PPi and NP-Pi + NP-PPi treatments were found in the case of $S$. marcescens over the tested nanoparticle concentration range (Figures 4C,D). Similar to the findings obtained for $P$. aeruginosa, a bactericidal effect was noted with concentrations greater or equal to $2 \%$ (w/vol) NP-PPi for E. faecalis (Figure 4E). No bactericidal effects were observed in the case of the combination treatment or with NP-Pi over the $1-10 \%$ (w/vol) nanoparticle range for E. faecalis (Figure 4F). Thus, the MIC value for NP-PPi was taken to be $\geq 2 \%$ $(\mathrm{w} / \mathrm{vol})$ and a total nanoparticle concentration of $1 \%(\mathrm{w} / \mathrm{vol})$ was used in all subsequent studies involving collagenase and biofilm production.

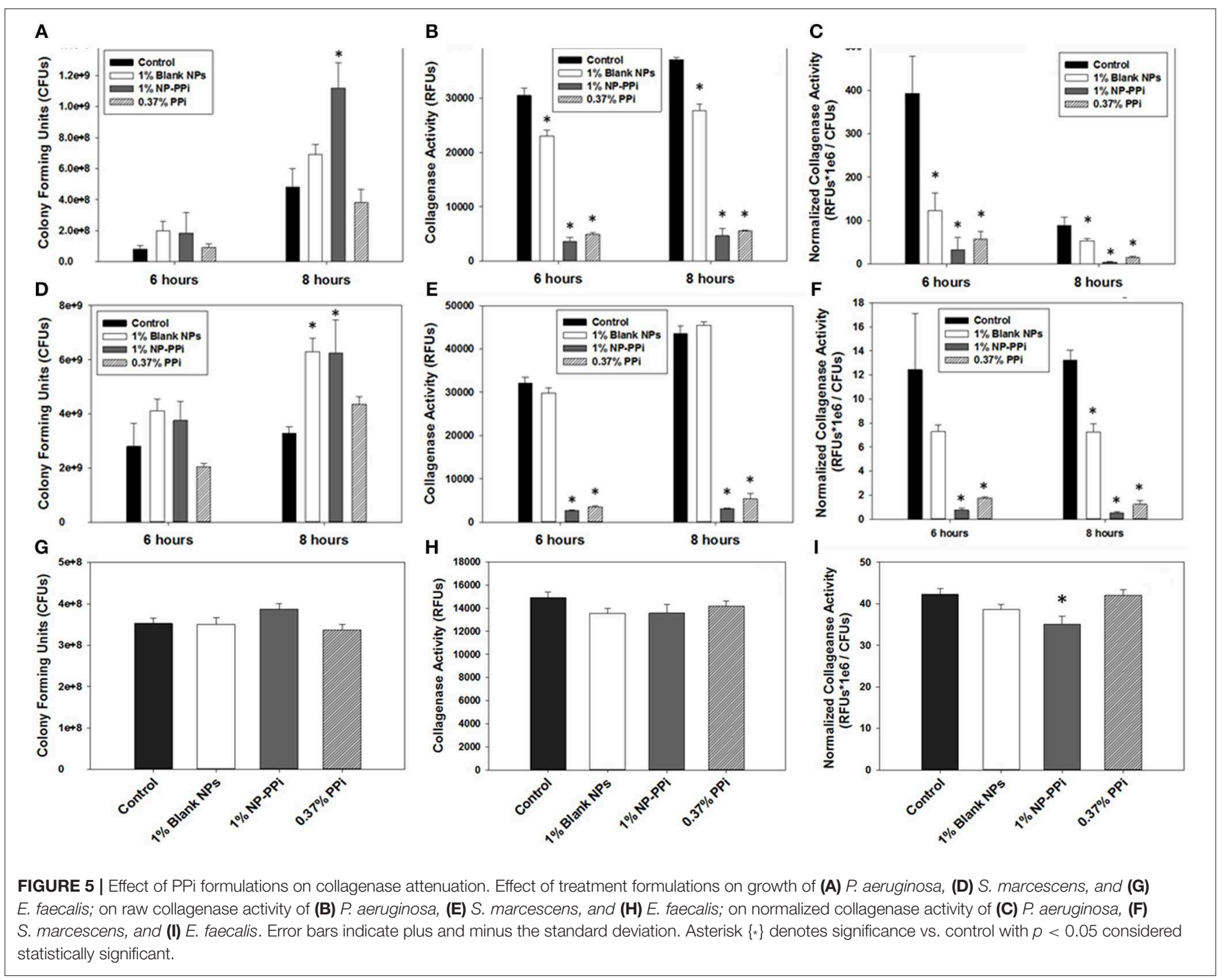




\section{Effect of Nanoparticle Formulations on Collagenase Activity and Growth}

Common antibiotics to prevent infection during intestinal surgery often indiscriminately eliminate the normal flora. This approach allows pathogenic bacteria to "bloom" and become antibiotic resistant. To determine the effectiveness of NP-PPi in maintaining bacterial survival, growth was quantified in the presence and absence of PPi treatment. The experimental groups included the no-treatment control, and treatment groups with blank NPs and NP-PPi (both at $1 \% \mathrm{w} / \mathrm{vol}$ ) and equivalent free PPi $(0.37 \% \mathrm{w} / \mathrm{vol})$. Negative controls without nanoparticles were also included to confirm that the presence of NPs did not interfere with bioluminescence or fluorescence measurements for quantifying bacterial growth. Both blank NPs and NP-PPi were found to promote $P$. aeruginosa growth at $6 \mathrm{~h}$ with statistical increases at $8 \mathrm{~h}$ as Figure 5A. In terms of raw collagenase values, the presence of PPi and NP-PPi results in significant attenuation of collagenase activity at both 6 and $8 \mathrm{~h}$ (Figure 5B). A significant drop in normalized collagenase is observed for all groups at the 8-h time point as compared to the 6-h time point (Figure 5C) which is attributed to the observed increases in $P$. aeruginosa growth at $8 \mathrm{~h}$ (Figure 5A). Overall, an over 90\% reduction in collagenase is observed as compared to the no treatment control at both time points (Figure 5C). While decreases in raw collagenase levels due to blank NP treatment are indicated, the presence of supplementation with NP-PPi and equivalent PPi result in statistical attenuation of collagenase activity of $P$. aeruginosa, with both treatments attaining at least a 6-fold decrease in normalized collagenase levels.

For S. marcescens, similar effects to those observed for $P$. aeruginosa occurred with increases in growth from 6 to $8 \mathrm{~h}$ (Figure 5D). While NP-PPi and PPi treatments significantly attenuated $S$. marcescens collagenase activity, the presence of blank NP had no effect on raw collagenase levels (Figure 5E). In terms of normalized collagenase, however, significant attenuation is observed with NP-PPi and PPi treatment, with an over $90 \%$ decrease in collagenase activity (Figure 5F) as compared to control. Although significant decreases in normalized collagenase levels with blank NP treatment was achieved at $8 \mathrm{~h}$, this effect may be attributed to the observed increase in growth (Figure 5D) as no statistical differences in raw collagenase levels for S. marcescens exist in the presence of blank NPs at the 6 or 8-h time point (Figure 5E). Thus, the data in Figure 5 demonstrate that NP-PPi and equivalent PPi treatments are effective at suppressing collagenase activity of gram-negative pathogens $(P$. aeruginosa and $S$. marcescens) and that treatment with blank NPPPi does not hinder their survival. In contrast to the observed responses observed with the gram-negative pathogens, blank NPs, NP-PPi, and free PPi were not as effective at attenuating collagenase levels of gram-positive E. faecalis (Figures $\mathbf{5 H}, \mathbf{I}$ ). In terms of normalized collagenase levels (Figure 5I), significant reductions in collagenase levels $(\sim 20 \%)$ are observed for the NPPPi treatment group as compared to control. The exact reason for the enhancement in collagenase attenuation achieved with NP$\mathrm{PPi}$ as compared to free PPi treatment is unknown. One possible explanation of these findings may be linked to quorum sensing, or to PEG interactions with the bacterial membrane. Nonetheless, these observations support the hypothesis of NP-PPi as an effective treatment for attenuation of collagenolytic activity, and most effective in the case of the gram-negative pathogens.

The inability of free PPi and consequently NP-PPi to attenuate collagenase activity of $E$. faecalis to the same extent as the gramnegative test pathogens is not entirely clear. It is known, however, that polyphosphate kinases (ppk1 and ppk2) are primarily responsible for synthesizing and hydrolyzing polyphosphates in bacteria. These enzymes, and high polyphosphate storage capacity have been confirmed in pathogens such as $P$. aeruginosa (Zhang et al., 2002; Racki et al., 2017), but recent studies have shown that the Enterococcus genus has a low genetic potential for accumulating and hydrolyzing polyphosphate (Breiland et al., 2018). Therefore, a possible explanation of our findings is that E. faecalis, does not have the capacity to readily break down
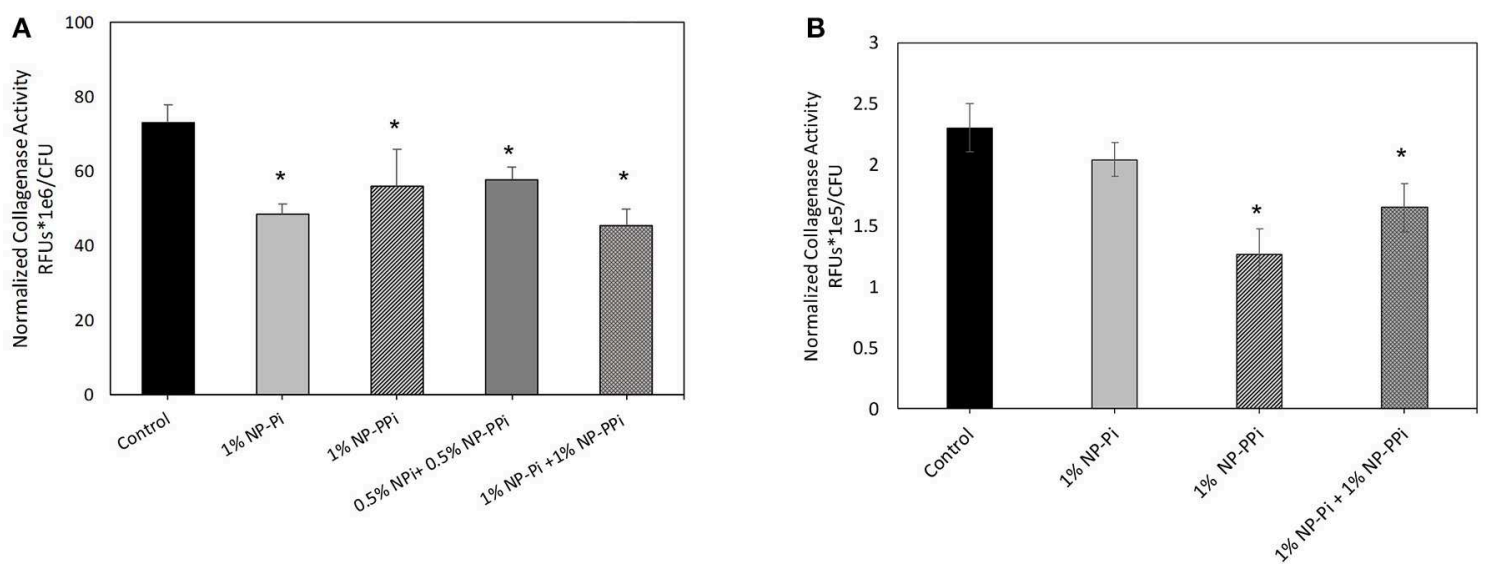

FIGURE 6 | Effect of NP-Pi, NP-PPi, and NP-Pi + NP-PPi mixture on collagenase attenuation of (A) E. faecalis and (B) P. aeruginosa. Data reported in terms of collagenase activity (RFUs) normalized to growth (CFUs). Error bars indicate plus and minus the standard deviation. Asterisk $\left\{{ }^{\star}\right\}$ denotes significance vs. control with $p<0.005$ considered statistically significant. 
PPi to phosphate to make it more readily bioavailable. Prior published studies indicate that oral administration of PPi does not promote healing in the surgically injured colon of mice intestinally inoculated with collagenolytic E. faecalis (pathogeninduced intestinal anastomotic leak) (Wiegerinck et al., 2018). Interestingly, a 20 -mer of phosphate (Pi-20) covalently attached to a high molecular weight linear PEG block copolymer was found to suppress in vitro collagenolytic activity and to enhance anastomotic healing in mice intestinally inoculated with collagenolytic E. faecalis (Wiegerinck et al., 2018). These findings suggest that while replenishment of phosphate levels at the injured host-pathogen interaction site are crucial to healing, a single form of phosphate may not be universally effective at attenuating collagenase expression. This indicates that delivery of different forms of phosphate may be required to confer broad spectrum efficacy across collagenolytic pathogens. To address this hypothesis, we investigated the effects of NP-Pi and the combination treatment (NP-Pi+NP-PPi) on collagenase attenuation of E. faecalis. The data in Figure 6A indicate that the $1 \% \mathrm{NP}-\mathrm{Pi}+1 \% \mathrm{NP}-\mathrm{PPi}$ combination yields the greatest attenuation of normalized E. faecalis collagenase production (38\% decrease) as compared to $1 \%$ (w/vol) NP-Pi (34\% decrease), $1 \%$ (w/vol) NP-PPi (23\% decrease), and a combination of 0.5 $\%$ N-PPi $+0.5 \%$ NP-Pi (22\% decrease) from the no treatment control. Importantly, the $1 \% \mathrm{NP}-\mathrm{Pi}+1 \% \mathrm{NP}-\mathrm{PPi}$ treatment significantly decreased $P$. aeruginosa collagenase production to a similar degree as NP-PPi alone. Finally, 1\% NP-Pi did not reduce $P$. aeruginosa collagenase production (Figure 6B). Combined, the data in Figure $\mathbf{6}$ demonstrate that the combination treatment involving sustained delivery of both $\mathrm{PPi}$ and $\mathrm{Pi}$ is most effective in attenuating collagenase production of the tested gram-positive and gram-negative pathogens. These findings are important as they form the basis for testing the efficacy of our approach in vivo and to further optimize the phosphate nanoparticle
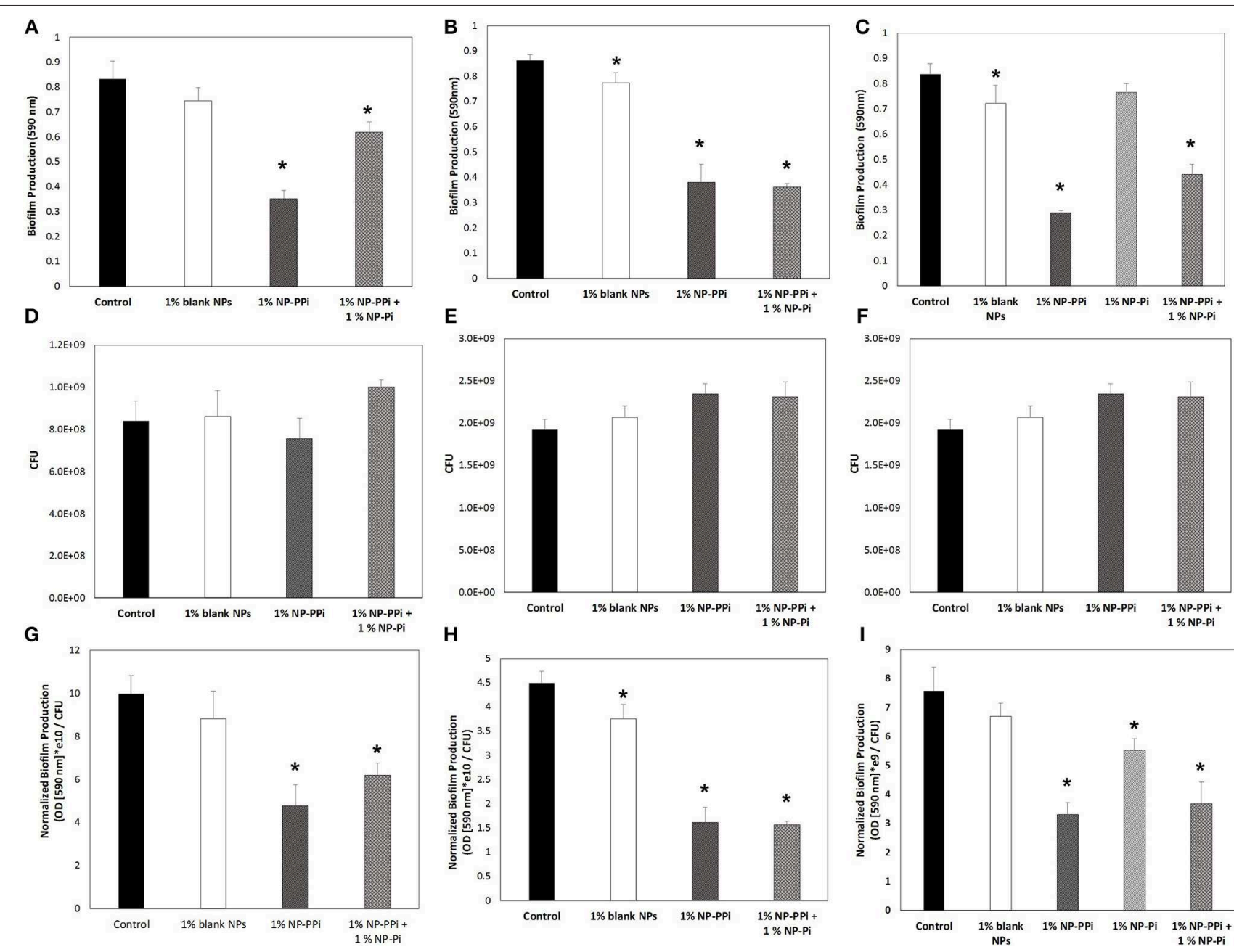

FIGURE 7 | Effect of nanoparticle formulations on biofilm attenuation of the tested pathogens. Raw biofilm levels shown for (A) P. aeruginosa, (B) S. marcescens, and (C) E. faecalis. CFU values shown for (D) P. aeruginosa, (E) S. marcescens, and (F) E. faecalis. Normalized biofilm values shown for (G) P. aeruginosa, (H) S. marcescens, and (I) E. faecalis. Error bars indicate plus and minus the standard deviation. Asterisk $\left\{{ }^{\star}\right\}$ denotes significance vs. control with $p<0.05$ considered statistically significant. 
combination treatment in animal models of intestinal injury and healing.

\section{Effect of NP-PPi Treatment on Biofilm Production}

Biofilm is a distinct pathogenic phenotype which plays a prominent role in tissue colonization and drug resistance. Therefore, the in vitro efficacy of sustained delivery of phosphates on biofilm production was also investigated. In previous studies we have shown that free PPi significantly attenuates biofilm production of $P$. aeruginosa and $S$. marcescens (Hyoju et al., 2017), however, the efficacy of NP-Pi, NP-PPi, or combinations of the two, on biofilm attenuation has not been previously explored.

Based on our observed findings involving collagenase attenuation (Figures 5, 6), we investigated whether similar treatments were effective against biofilm production. The data in Figure 7 indicate that for all test pathogens the 1\% NP$\mathrm{PPi}$ and $1 \%$ NP-Pi $+1 \%$ NP-PPi treatments significantly reduce raw (Figures 7A-C) and normalized (Figures 7G-I) biofilm production as compared to the no treatment control. In the case of $P$. aeruginosa, the reduction in normalized biofilm from the control was found to be $52 \%$ with NP-PPi and $38 \%$ with the combination treatment (Figure 7G). For S. marcescens attenuation in normalized biofilm between the two phosphate treatments was found to be similar (64 and $65 \%$ reduction for NP-PPi and NP-Pi + NP-PPi, respectively, from the control). Interestingly, the greatest reduction in E. faecalis biofilm production occurred with NP-PPi $(56 \%$ reduction) treatment, followed by the combination treatment (51\% reduction) and NP-Pi (27\% reduction) from the no treatment control (Figure 7I). These findings corroborate with those observed for collagenase attenuation, further validating our hypothesis that the NP-Pi + NP-PPi formulation provides broad spectrum treatment by attenuating collagenase and biofilm levels of gram-negative and gram-positive intestinal pathogens whose phenotype impairs healing.

\section{CONCLUSION}

In summary, PEG hydrogel nanoparticles have been synthesized for sustained release of monophosphate (sodium monobasic phosphate) and polyphosphate (sodium hexametaphosphate). Our findings indicate that a combination treatment of NP-Pi and NP-PPi was most effective at attenuating collagenase and biofilm production in clinically relevant strains of gram-negative $P$. aeruginosa and S. marcescens and gram-positive E. faecalis

\section{REFERENCES}

Breiland, A. A., Flood, B. E., Nikrad, J., Bakarich, J., Husman, M., Rhee, T., et al. (2018). Polyphosphate-accumulating bacteria: potential contributors to mineral dissolution in the oral cavity. Appl. Environ. Microbiol. 84, e2440e2417. doi: 10.1128/AEM.02440-17

Buffie, C. G., and Pamer, E. G. (2013). Microbiota-mediated colonization resistance against intestinal pathogens. Nat. Rev. Immunol. 13, 790-801. doi: $10.1038 /$ nri3535 expressing high collagenolytic activity. It is important to note that the nanoparticle formulations did not display bacteriostatic effects across the gram-negative and grampositive pathogens tested, which indicates their potential as an effective drug delivery approach to target virulence without eradicating the normal microbiome which is beneficial to healing. A variety of other phenotypes, not investigated in this study, contribute to bacterial virulence, including excessive production of elastases, proteases, alginates, rhamnolipids, and hemolysins. Our future efforts will focus on determining whether our proposed treatment using phosphate and/or polyphosphates and combinations thereof are also capable of attenuating these pathogenic phenotypes. Future studies will also utilize reporter strains of the test pathogens to quantify the efficacy of the proposed treatment on quorum sensing suppression. Finally, our current efforts focus on evaluating the mucoadhesiveness of the proposed Pi and PPi nanoparticle formulations on explants of intestinal injury and their preclinical efficacy in accelerating healing in murine model of intestinal injury.

\section{DATA AVAILABILITY}

All datasets generated for this study are included in the manuscript and/or the Supplementary Files.

\section{AUTHOR CONTRIBUTIONS}

DN, OZ, SHH, SKH, FT, JA, and GP contributed conception and design of the study. DN, MP, SHH performed the experiments. DN and MP performed the statistical analysis. DN wrote the first draft of the manuscript. DN, MP, FB, and GP wrote sections of the manuscript. All authors contributed to manuscript revision, read, and approved the submitted version.

\section{FUNDING}

This work was financially supported by the National Institutes of Health Grant numbers R21AI124037-01 (awarded to GP, PI) and R01-GM062344-18 (awarded to JA, PI).

\section{SUPPLEMENTARY MATERIAL}

The Supplementary Material for this article can be found online at: https://www.frontiersin.org/articles/10.3389/fbioe. 2019.00153/full\#supplementary-material Infect. Control. 1, 1-39. doi: 10.1186/2047-2994-1-39

Fink, D., Romanowski, K., Valuckaite, V., Babrowski, T., Kim, M., Matthews, J. B., et al. (2011). Pseudomonas aeruginosa potentiates the lethal effect of intestinal ischemia-reperufsion injury: the role of in vivo virulence activation. J. Trauma 71, 1575-1582. doi: 10.1097/TA.0b013e318 $21 \mathrm{cb} 7 \mathrm{e} 5$

Harold, F. M. (1966). Inorganic polyphosphates in biology: structure, metabolism, and function. Bacteriol. Rev. 30, 772-794. 
Hyoju, S. K., Klabbers, R. E., Aaron, M., Krezalek, M. A., Zaborin, A., Wiegerinck, M., et al. (2017). Oral polyphosphate suppresses bacterial collagenase production and prevents anastomotic leak due to Serratia marcescens and Pseudomonas aeruginosa. Ann. Surg. 267, 1112-1118. doi: 10.1097/SLA.0000000000002167

Karsi, A., and Lawrence, M. L. (2007). Broad host range fluorescence and bioluminescence expression vectors for Gram-negative bacteria. Plasmid 57, 286-295. doi: 10.1016/j.plasmid.2006.11.002

Kumble, K. D., and Kornberg, A. (1995). Inorganic polyphosphate in mammalian cells and tissues. J. Biol. Chem. 270, 5818-5822. doi: 10.1074/jbc.270.11.5818

Lorenz, B., Münkner, J., Oliveira, M. P., Kuusksalu, A., Leitão, J. M., Müller, W. E., et al. (1997). Changes in metabolism of inorganic polyphosphate in rat tissues and human cells during development and apoptosis. Biochim. Biophys. Acta 1335, 51-60. doi: 10.1016/S0304-4165(96)00121-3

Luong, P. M., Shogan, B. D., Zaborin, A., Belogortseva, N., Shrout, J. D., Zaborina, O., et al. (2014). Emergence of the P2 phenotype in Pseudomonas aeruginosa $\mathrm{PAO} 1$ strains involves various mutations in mexT or mexF. J. Bacteriol. 196, 504-513. doi: 10.1128/JB.01050-13

Ohigashi, S., Sudo, K., Kobayashi, D., Takahashi, T., Nomoto, K., and Onodera, H. (2013). Significant changes in the intestinal environment after surgery in patients with colorectal cancer. J. Gastrointest. Surg. 17, 1657-1664. doi: 10.1007/s11605-013-2270-x

Olivas, A. D., Shogan, B. D., Valuckaite, V., Zaborin, A., Belogortseva, N., Musch, M., et al. (2012). Intestinal tissues induce an SNP mutation in Pseudomonas aeruginosa that enhances its virulence: possible role in anastomotic leak. PLoS ONE 7:e44326. doi: 10.1371/journal.pone.0044326

Racki, L. R., Tocheva, E. I., Dieterle, M. G., Sullivan, M. C., Jensen, G. J., and Newman, D. K. (2017). Polyphosphate granule biogenesis is temporally and functionally tied to cell cycle exit during starvation in Pseudomonas aeruginosa. Proc. Natl. Acad. Sci. U.S.A. 114, E2440-E2449. doi: 10.1073/pnas.1615575114

Rao, N. N., Gómez-García, M. R., and Kornberg, A. (2009). Inorganic polyphosphate: essential for growth and survival. Annu. Rev. Biochem. 78, 605-647. doi: 10.1146/annurev.biochem.77.083007.093039

Shakhsheer, B. A., Versten, L. A., Luo, J. N., Defazio, J. R., Klabbers, R., Christley, S., et al. (2016). Morphine promotes colonization of anastomotic tissues with collagenase - producing Enterococcus faecalis and causes leak. J. Gastrointest. Surg. 20, 1744-1751. doi: 10.1007/s11605-016-3237-5

Shogan, B. D., Belogortseva, N., Luong, P. M., Zaborin, A., Lax, S., Bethel, C., et al. (2015). Collagen degradation and MMP9 activation by Enterococcus faecalis contribute to intestinal anastomotic leak. Sci. Transl. Med. 7:286ra268. doi: 10.1126/scitranslmed.3010658

Vadlamudi, S., Nichols, D., Papavasiliou, G., and Teymour, F. (2019). Phosphate-loaded hydrogel nanoparticles prepared via inverse miniemulsion polymerization. Macromol. React. Eng. 13:1800066 doi: 10.1002/mren.201800066

Valuckaite, V., Zaborina, O., Long, J., Hauer-Jensen, M., Wang, J., Holbrook, C., et al. (2009). Oral PEG 15-20 protects the intestine against radiation: role of lipid rafts. Am. J. Physiol. Gastrointest. Liver Physiol. 297, G1041-G1052. doi: 10.1152/ajpgi.00328.2009

Wiegerinck, M., Hyoju, S. K., Mao, J., Zaborin, A., Adriaansens, C., Salzman, E., et al. (2018). Novel de novo synthesized phosphate carrier compound ABAPEG20k-Pi20 suppresses collagenase production in Enterococcus faecalis and prevents colonic anastomotic leak in an experimental model. Br. J. Surg. 105, 1368-1367. doi: 10.1002/bjs.10859

Yin, Y., Papavasiliou, G., and Zaborina, O. (2017). De novo synthesis and functional analysis of polyphosphate-loaded Poly(ethylene) glycol hydrogel nanoparticle targeting pyocyanin and pyoverdin production in Pseudomonas aeruginosa as a model intestinal pathogen. Ann. Biomed. Eng. 45, 1058-1068. doi: 10.1007/s10439-016-1740-1

Zaborin, A., Gerdes, S., Holbrook, C., Liu, D. C., Zaborina, O. Y., and Alverdy, J. C. (2012). Pseudomonas aeruginosa overrides the virulence inducing effect of opioids when it senses an abundance of phosphate. PLOS ONE 7:e34883. doi: 10.1371/journal.pone.0034883

Zaborin, A., Romanowski, K., Gerdes, S., Holbrook, C., Lepine, F., Long, J., et al. (2009). Red death in Caenorhabditis elegans caused by Pseudomonas aeruginosa PAO1. Proc. Natl. Acad. Sci. U.S.A. 106, 6327-6332. doi: 10.1073/pnas.0813199106

Zaborin, A., Smith, D., Garfield, K., Quensen, J., Shakhsheer, B., Kade, M., et al. (2014). Membership and behavior of ultra-low diversity pathogen communities present in the gut of humans during prolonged critical illness. MBio 5, e01361e01314. doi: 10.1128/mBio.01361-14

Zhang, H., Ishige, K., and Kornberg, A. (2002). A polyphosphate kinase (PPK2) widely conserved in bacteria. Proc. Natl. Acad.Sci. U.S.A. 99, 16678-16683. doi: $10.1073 /$ pnas.262655199

Conflict of Interest Statement: The authors declare that the research was conducted in the absence of any commercial or financial relationships that could be construed as a potential conflict of interest.

Copyright (c) 2019 Nichols, Pimentel, Borges, Hyoju, Teymour, Hong, Zaborina, Alverdy and Papavasiliou. This is an open-access article distributed under the terms of the Creative Commons Attribution License (CC BY). The use, distribution or reproduction in other forums is permitted, provided the original author(s) and the copyright owner(s) are credited and that the original publication in this journal is cited, in accordance with accepted academic practice. No use, distribution or reproduction is permitted which does not comply with these terms. 